\title{
LETRAMENTO EM SAÚDE NA PESSOA IDOSA EM TEMPOS DE PANDEMIA E INFODEMIA DO COVID-19: UM DESAFIO MUNDIAL
}

\author{
Rachel da Silva Serejo Cardoso' \\ ORCID: 0000-0002-7283-8086 \\ Juliana Mineu Pereira" \\ ORCID: 0000-0001-8509-9174
}

Allison Barros Santana"II

ORCID: 0000-0002-9795-7663

Selma Petra Chaves Sá'

ORCID: 0000-0001-9878-7179

Mirian da Costa Lindolpho'

ORCID: 0000-0002-2503-4827

Miriam Marinho Chrizostimo' ORCID: 0000-0001-7498-4637

Rosimere Ferreira Santana' ORCID: 0000-0002-4593-3715

\footnotetext{
IUniversidade Federal Fluminense. Niterói, Rio de Janeiro, Brasil.

"Universidade Estadual do Ceará. Fortaleza, Ceará, Brasil.

"'Universidade Federal do Tocantins. Palmas, Tocantins. Brasil.
}

Autor Correspondente: Rachel da Silva Serejo Cardoso E-mail: rachelserejo@gmail.com

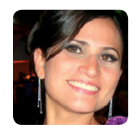

Como citar:

Cardoso RSS, Pereira JM, Santana AB, Sá SPC

Lindolpho MC, Chrizostimo MM, Santana RF. Letramento em saúde na pessoa idosa em tempos de pandemia e infodemia do covid-19: um desafio mundial. In: Santana RF (Org.). Enfermagem gerontológica no cuidado do idoso em tempos da COVID 19. Brasilia, DF: Editora ABen; 2021.171 p. (Serie Enfermagem e Pandemias, 5). https://doi.org/10.51234/aben.21.e05.c21

\section{INTRODUÇÃO}

Atualmente, vive-se em tempos de pandemia sendo difícil a convivência entre os indivíduos, a família e a sociedade. Diante do rápido desenvolvimento da doença do novo coronavírus (SARS-CoV-2) em todo o mundo, demandou das pessoas a necessidade de adequação do comportamental que dever ser tão rápido quanto a evolução da doença e, tal situação, atingiu fortemente os idosos por ser grupo de risco $^{(1)}$. Esta vulnerabilidade se dá pelo o próprio processo fisiológico do envelhecimento que incita a diminuição da eficácia do sistema imune, aumentando propensão a morbidade e mortalidade de doenças infecciosas ${ }^{(2)}$.

Como medida para evitar a propagação ou disseminação da infecção da doença em todo o mundo, o isolamento social e a comunicação em saúde foram as principais ações estratégicas equânimes e condizentes em todos os países frente à pandemia da COVID-19. O maior objetivo nesta comunicação globalizada e rápida, está em educar as pessoas sobre o coronavírus, da síndrome respiratória aguda grave (SARS-CoV-2) e como evitar a propagação ou disseminação da infecção, oferecendo medidas simples e práticas como lavar as mãos, manter a distância física, isolamento social e até mesmo onde encontrar informações e recomendações recentes ${ }^{(1)}$.

Como exemplo dos meios de comunicação em saúde, a Organização Mundial de Saúde (OMS) lançou nova plataforma de informação chamada OMS Rede de Informação para Epidemias (EPI-WIN), com o objetivo de usar uma série de amplificadores para compartilhar sob medida, informações com grupos-alvo específicos ${ }^{(3)}$. Contudo, apesar da informação em saúde se tratar de uma das estratégias amplamente difundidas para o controle do novo coronavírus, que causa a COVID 19, surge um novo problema, concomitante a este evento, que é a infodemia ${ }^{(1)}$.

A infodemia evidencia o analfabetismo em saúde da população, tendo em vista que no meio de uma avalanche 
de informações em saúde, existem divulgação de informações complexas, contraditórias, boatos, notícias falsas e as chamadas "Fake News" que, muitas vezes, vão mais rápido e mais longe que o próprio vírus. Sendo assim, está sendo um desafio constante para a OMS e uma luta diária, não só com a pandemia do COVID-19, mas sobretudo, com a infodemia(1).

Perante o fenômeno da infodemia, nos tempos atuais, nunca foi tão urgente, o letramento em saúde principalmente, no que concerne um grupo vulnerável que são os idosos. A capacidade dos indivíduos de obter uma informação, compreender as razões por trás das recomendações e refletir nos resultados e em ações possíveis, impactando diretamente na tomada de decisão é de grande responsabilidade social, inclusive do ponto de vista ético, levando em consideração que a disseminação do vírus, depende de atitudes individuais que impactam na população geral, quiçá nos grupos de risco ${ }^{(1)}$.

No mês de abril de 2020, saiu uma nota na revista Lancet, revista de grande relevância e reconhecida pelos seus artigos científicos, onde em sua publicação destaca-se que, o letramento em saúde na COVID-19 é um problema que ainda está subestimado. Nesse sentido, esta mesma publicação aponta que, o desenvolvimento do letramento em saúde é mais atual e necessário do que nunca para preparar os indivíduos para situações que exigem reações rápida. $\mathrm{O}$ artigo ainda ressalta que, deve ser visto como uma responsabilidade social e também à solidariedade tanto para as pessoas que precisam de informações e serviços quanto para os indivíduos que as fornecem e garantem sua acessibilidade para a população em geral ${ }^{(1)}$.

Considera-se que tal informação é primordial para a população idosa já acometida por tantas doenças e cormobidades e ainda mais, diante de uma situação uma doença tão perigosa para os idosos tendo em vista as complicações da mesma. Diante da relevância do letramento em saúde na pessoa idosa a nível global no âmbito coletivo e não apenas individual e em sua magnitude, nos motivou a refletir esta temática, tecendo contribuições para a enfermagem gerontológica.

\section{OBJETIVO}

Refletir sobre a importância do letramento em saúde para a pessoa idosa frente à Pandemia e infodemia do COVID-19 no contexto mundial e suas implicações para enfermagem gerontológica.

\section{MÉTODOS}

Trata-se de um estudo teórico-reflexivo que aborda sobre o desafio mundial do letramento em saúde para a pessoa idosa em tempos de pandemia e infodemia da COVID-19, e aponta implicações para enfermagem gerontológica.

As reflexões foram embasadas em artigos internacionais e nacionais relacionados ao tema. Assim, as considerações foram feitas em 4 etapas, todas na perspectiva da informação da pessoa idosa diante da pandemia por coronavírus a saber: 1) letramento em saúde na pessoa idoso como grupo de risco SARS-COV-2; 2) a pessoa idosa em tempos de pandemia da COVID-19- o letramento e a infodemia; 3) os cuidadores e os familiares da pessoa idosa - impacto do letramento 4) ações de letramento na enfermagem gerontológicapossibilidades de avanços para a pessoa idosa.

\section{Letramento em saúde na pessoa idosa como grupo de risco}

A consequência dos baixos índices de Letramento em Saúde traz prejuízo a saúde aos indivíduos. O conhecimento insuficiente sobre doenças, bem como baixa adesão as medidas de prevenção e promoção e baixa adesão aos medicamentos, acarreta risco a saúde, principalmente no que tange no momento de uma pandemia. Tais situações elevam os custos de saúde, pois promovem maior hospitalização. Uma parcela da população que é mais afetada por este cenário são os idosos ${ }^{(4)}$. 
A população tende a ser mais propensa a obter pouco acesso a informações de saúde, consequentemente afetando em seu autocuidado, principalmente aqueles com baixa escolaridade. E dentre estes com piores índices de escolaridades são os que exibem maior necessidades de cuidados de saúde, pois apresentam maiores índices de doenças crônicas, pior funcionamento físico e pior declínio na saúde mental(5).

Além das comorbidades frequentes ao idoso, o mesmo enfrenta uma condição de saúde aguda na pandemia do COVID-19, por estar na lista da população de risco. A COVID-19 é uma condição aguda, grave, que necessita de cuidados preventivos para não disseminação do vírus. A infecção pela COVID-19 nesta população é marcada por uma intensa tempestade de citocinas inflamatórias o que pode levar a síndrome da angústia respiratória aguda grave, uma condição que eleva a taxa de mortalidade especificamente nesta população(4).

Por conseguinte, o letramento em saúde no idoso frente a pandemia da COVID-19 é desafiador para os profissionais de saúde, cuidadores e familiares. A avalanche de informações, muitas vezes errôneas causam confusão no idoso. O mesmo fica sem saber qual orientação seguir, ficando vulnerável a aplicar cuidados de saúde que o tornam potenciais infectados pela doença.

\section{A pessoa idosa em tempos de pandemia da COVID-19 - 0 letramento e a infodemia}

Em meio de uma pandemia, ainda existem pessoas que ignoram as políticas do COVID-19, negligenciando as precauções estabelecidas pelos órgãos da saúde pública, mostrando-nos um comportamento irracional, motivados muitas vezes pela percepção equivocada de risco, permitindo os chamados freeriders, este grupo de pessoas, ignoram pedido de comportamento protetor e preventivo, sentindo uma falsa sensação de invulnerabilidade, causando uma injustiça, principalmente para os idosos que são grupos de risco $^{(3)}$.

Nesse sentido que a OMS se reuniu em Geneva nos dias 7 e 8 de abril de 2020, através da Rede de Informação da OMS para Epidemias (EPI-WIN). Foi feito uma consulta on-line global de dois dias sobre o gerenciamento da infodemia de COVID-19. O objetivo desta consulta foi reunir fontes de ideias para combater o infodêmico, desenvolver uma estrutura de resposta infodêmica para orientar intervenções direcionadas para promover a disseminação de informações confiáveis sobre o COVID-19 e reduzir desinformação, boatos e mitos sobre o COVID-19(6).

Esse encontro contou com um grupo interdisciplinar de especialistas e dos 1.300 participantes do webinar. Juntamente com as sugestões dos palestrantes, mais de 350 ideias foram enviadas por meio de um fórum interativo on-line durante a consulta. Juntos, tudo isso formará a base para uma estrutura infodêmica do COVID-19 para orientar as ações que governos e instituições de saúde pública podem adotar ${ }^{(6)}$.

O resumo da reunião sobre a estrutura da gestão infodêmica, se dá por quatro pilares identificados pelo acrônimo ISAQ. Sendo I - identifyevidence, traduzindo para o português consecutivamente, identificar as evidências, S- Simplify Knowledge, simplificar o conhecimento, A-amplifyaction, amplificar a ação e Q- Quanitify impacto, quantificar o impacto(6).

A estrutura de resposta infodêmica consistirá baseado na discussão e proposta dos participantes para intervenções em cada pilar citado. A saber: Identificar evidências: digitalizar, analisar e verificar evidências e informações, Simplificar o conhecimento: maneiras de interpretar e explicar a ciência para diferentes públicos, Ampliar ação: tanto no alcance e em ouvir as preocupações do público e fornecer conselhos para ação, definir melhores e novos canais de distribuição e Quantificar o impacto: descrever o infodêmico, mensurar a mudança e o impacto do discurso digital; informar intervenções a nível global, nacional e local e informar os Estados-Membros engajamento em um “diálogo social” mais coordenado(6).

Nesse sentido, a OMS planeja atividades de curto e médio prazo, fluxos de trabalho e acompanhamentos fortalecido e estabelecido para a coordenação, comunicação e construção da comunidade de práticas, métodos e medidas para informar políticas e ações e medir o impacto, estruturas e métodos de monitoramento à "infodemiologia", análise de informações digitais e ferramentas, análise e percepções do contexto sócio-comportamental e cultural, gerenciamento de verificação de fatos e desinformação, identificar evidências, 
simplificando o conhecimento, ampliar as informações e atividades para que as informações direcionadas cheguem aos indivíduos ${ }^{(6)}$.

Como já vimos, a infodemia é a superabundância de informações, algumas precisas e outras que não ocorrem durante uma pandemia. Como patógenos em epidemias, a desinformação se espalha mais e mais rapidamente e adiciona complexidade à resposta a emergências de saúde. Para dar continuidade à discussão proposta pela OMS para com vista à planejar ações coordenadas, foi proposto a Pré-conferência para o dia 29 de junho de 2020 a $1^{\text {a }}$ Conferência de Infodemiologia da OMS. Na pré-conferência, os especialistas se envolvem com o público com 7 palestras sobre como a infodemia afeta o mundo atualmente e seu reflexo e como pode ser gerenciado(6).

Nesta perspectiva, entendendo a importância do letramento em saúde relacionada à pandemia da COVID-19, que um grupo de pesquisadores da Universidade de Medicina de Duke-NUS em Singapura iniciou em 07 de junho a primeira revisão sistemática na temática. O objetivo desta pesquisa foi em resumir a avaliação, os níveis e os determinantes da alfabetização em saúde relacionada à pandemia e seus resultados clínicos associados $^{(7)}$.

A pesquisa mencionada conclui que, em geral, o nível de alfabetização em saúde relacionada à pandemia permanece sub-ideal entre os médicos e população não médica. Isso é preocupante, dado o papel crítico da alfabetização em saúde na redução da disseminação do contágio e mitigação dos efeitos das pandemias. Existe uma necessidade urgente de desenvolver questionários atualizados, validados e padronizados para a avaliação rápida da saúde relacionada à pandemia alfabetização(7).

Determinantes importantes associados a melhores níveis de conhecimento em saúde, como idade avançada, gênero feminino, status de emprego e nível de educação foram destacados nesta revisão, desta forma, podemos concluir que o idoso é um fator de vulnerabilidade para analfabetização em saúde ${ }^{(7)}$.

Cuidados de saúde, administradores e formuladores de políticas precisam estar atentos a esses determinantes ao formular disseminação de informações e intervenções críticas relacionadas a pandemias para melhorar a alfabetização em saúde da população. Nota-se nesta pesquisa também que são necessários mais estudos para avaliar os resultados clínicos associados à pandemia alfabetização relacionada à saúde ${ }^{(7)}$.

\section{Os cuidadores e os familiares da pessoa idosa - Impacto do letramento}

Os cuidadores e familiares desempenham um papel fundamental na vida dos idosos, no que se refere ao contexto de suas atividades de vida diária ${ }^{(8)}$. Essa situação se configura como essencial no atual cenário mundial, tendo em vista a pandemia do novo coronavírus. O domínio das habilidades de leitura e compreensão das informações precisam ser trabalhadas para não afetar negativamente a saúde do idoso, principalmente aquelas relacionadas ao ensino do autocuidado.

Levando em consideração o aparecimento das doenças crônicas nos idosos e sua relação à COVID-19, os desafios de autocuidado às atividades cotidianas de controle dos sintomas, cumprimento ao regime terapêutico, mudanças fisiológicas da idade na sua composição física e a alternância do estilo de vida para lidar com as limitações advindas das doenças crônicas. Isso faz do Letramento em Saúde um forte aliado no gerenciamento do autocuidado e, fortuitamente, um enfrentar positivo em época de pandemia ${ }^{(9)}$, vendo nos cuidadores e familiares um elo no processo de cuidado da saúde, segurança e independência.

O Letramento em Saúde para os cuidadores e familiares de idosos, deve ser considerado, neste contexto, posto auxiliar na comunicação e na ajuda ao acesso à informação que o idoso necessita para executar seu plano terapêutico. Estudos evidenciam a importância de considerar o letramento dos cuidadores e familiares, tendo em vista as muitas tarefas pelas quais estes se tornam responsáveis, incluindo assistência nas atividades cotidianas com medicamentos, apoio médico e também a tomada de decisões. Quando faltam as habilidades necessárias, várias consequências podem surgir em termos de resultados de saúde das pessoas idosas ${ }^{(9)}$. 
Neste sentido, foi desenvolvido o TOFHLA - Teste de Alfabetização Funcional em Saúde em Adultos, para medir as habilidades de leitura e compreensão em situações comumente encontradas no sistema de saúde. Recentemente foi traduzido e adaptado o S-TOFHLA no Brasil, tendo sido amplamente utilizado em populações com idade avançada ${ }^{(9)}$.

Vale ressaltar que o S-TOFHLA foi utilizado em pesquisa recente na cidade de São Paulo e trouxe dados que requerem bastante atenção. $27 \%$ dos cuidadores avaliados apresentavam níveis inadequados de alfabetização em saúde. Uma proporção maior de indivíduos com baixa escolaridade apresentou alfabetização em saúde inadequada e limítrofe. Aproximadamente 1/3 dos cuidadores apresentaram níveis limítrofes e inadequados de alfabetização em saúde ${ }^{(9)}$. Esses resultados destacam as dificuldades de muitos cuidadores em compreender informações sobre saúde.

Reitera-se que o nível de Letramento em Saúde afeta diretamente a capacidade do indivíduo em atuar no seu contexto de vida. Melhorar o acesso às informações, sua interpretação e atuação prática, é uma das ferramentas capazes de alcançar melhores resultados em saúde ${ }^{(9)}$, neste sentido, cabe ao Enfermeiro direcionar sua atenção a essa população, entendendo que a estratégia de ensino, como o Letramento, pode resultar em melhoria da qualidade da assistência e a dinâmica do autocuidado.

\section{Ações de letramento na enfermagem gerontológica - Possiblidades de avanço para a pessoa idosa}

Os idosos têm taxas mais alta de baixo nível de alfabetização em saúde em comparado com o resto da população. O baixo nível de alfabetização em saúde nesta população crescente está associado a maiores taxas de hospitalização, menor uso de serviços preventivos, menos conhecimento sobre medicamentos, aumento da mortalidade, incapacidade de controlar doenças crônicas e baixo estado de saúde autorreferido ${ }^{(10)}$.

Devemos desenvolver estratégias de ensino adequadas à idade e planejar de forma clara e objetiva informações necessárias e específicas para cada indivíduo, sem deixar de levar em considerações intervenções de ensino a esses idosos, que compensam os efeitos cognitivos, sensoriais e físicos do envelhecimento, ao mesmo tempo que promova as independências ${ }^{(10)}$.

Nesse sentindo, é necessário compreender que novos conhecimentos ou habilidades devem ser vinculados a experiências anteriores para ajudar os idosos a conectar as experiências vividas para facilitar a nova aprendizagem, sendo necessário criar um ambiente que encoraja os idosos a manter ativos em seus cuidados em saúde, sempre salientando a manutenção de sua independência, desta forma, o enfermeiro deve ser um facilitador, permitindo o idoso apreender no seu tempo de forma lenta e deliberada ${ }^{(10)}$.

Além de estratégias adequadas à idade, os enfermeiros, devem observar pistas sutis que sugerem baixo nível de letramento em saúde, como não ter óculos de leitura disponíveis, apresentar um comportamento incomum como por exemplo de agressividade quando solicitado para revisar alguma informação(10).

Nesse sentido, as principais implicações importantes para enfermagem, inicia-se na formação dos futuros enfermeiros, com proposta de inclusão curricular, onde os alunos deverão saber avaliar e intervir com a pessoa idosa com baixo letramento em saúde. Sabemos que a educação em saúde é de responsabilidade central da enfermagem. Desta forma, essa reflexão sugere que os estudantes de enfermagem devem ser capazes de avaliar os pacientes quanto às limitações em letramento em saúde, e garantir intervenções adequadas, para isso, é imperativo que os enfermeiros sejam qualificados para comunicar com o paciente com baixa alfabetização em saúde adequadamente ${ }^{(10)}$.

Levando em consideração os desafios significativos no processo de comunicação com os idosos, é importante salientar que os enfermeiros gerontológicos devem ser proativos na promoção de uma comunicação clara e individualizada para aumentar a chance de ser compreendido pelos idosos, criar um ambiente acolhedor, comunicar de maneira centrada no paciente, reforçar a palavra falada, verificar o entendimento, provendo sempre uma comunicação objetiva e clara. Sugere-se que os enfermeiros precisam se apropriar desta responsabilidade profissional, ética e legal de saber comunicar informações aos idosos de forma que eles podem compreender ${ }^{(10)}$. 


\section{CONSIDERAÇÕES FINAIS}

A prevenção da COVID-19 depende das ações estruturadas pelos órgãos oficiais em saúde, mas também do autocuidado que cada pessoa realiza em seu favor e o mantém nos contextos de relações sociais. E essas ações de prevenção necessitam ser conhecidas para que sua implementação alcance seus objetivos. Frente à essa demanda o baixo letramento em saúde na pessoa idosa é mais um fator a torná-la como vulnerável, além das condições proporcionadas pelo envelhecimento. Assim, a identificação do baixo Letramento da pessoa idosa e as ações elaboradas pelos enfermeiros contribuem com a instrumentalização, promovem a saúde e empodera o idoso para o enfretamento da pandemia. Considera-se que o Letramento em Saúde abre-se como a possibilidade de propiciar um autocuidado seguro à pessoa idosa não só para o enfrentamento da pandemia do COVID-19, mas para todas as situações nas quais ele precisará tomar uma decisão referente a sua saúde.

\section{AGRADECIMENTO}

Agradecimento ao Departamento Científico de Enfermagem Gerontológica da ABEn Nacional.

\section{REFERÊNCIAS}

1. Paakkari L, Okan O. COVID-19: health literacy is an underestimated problem. Lancet Public Health. 2020;5(5):e249-e250. https://doi.org/10.1016/S2468-2667(20)30086-4

2. Argenta C, Nunes DP, Hammerschimidt KSA, Niwa LMS, Souza PA, Melo POC. Distanciamento social do idoso saudável durante a pandemia COVID-19: possibilidades e desafios. In: Enfermagem gerontológica no cuidado do idoso em tempo da COVID 19 [Internet]. Brasília, DF: ABEn; 2020[cited 2020 Jun 29]. 74 p.(Série Enfermagem e Pandemias). Available from: http://www.abennacional.org.br/site/wp-content/uploads/2020/05/E-BOOK-GERONTO.pdf

3. Zarocostas J. “How to fight an infodemic." Lancet. 2020;395(10225):676. https://doi.org/10.1016/S0140-6736(20)30461-X

4. Meftahi GH, Jangravi Z, Sahraei H, Bahari Z. The possible pathophysiology mechanism of cytokine storm in elderly adults with COVID-19 infection: the contribution of "inflame-aging". Inflamm Res. 2020;11:1-15. https://doi.org/10.1007/ s00011-020-01372-8

5. Verney SP, Gibbons LE, Dmitrieva NO. Health literacy, sociodemographic factors, and cognitive training in the active study of older adults. Int J Geriatr Psychiatry. 2019;34(4):563-70. https://doi.org/10.1002/gps.5051

6. World Health Organization (WHO). Pre-conference: 1st WHO Infordemiology Conference [Internet]. 2020[cited 2020 Jun 29]. Available from: https://www.who.int/news-room/events/detail/2020/06/29/default-calendar/pre-conference-1st-whoinfodemiology-conference (WHO,

7. Seng JJB, Yeam CT, Huang WC, Tan NC, Low LL. Pandemic related health literacy: a systematic review of literature in COVID-19, SARS and MERS pandemics. 2020:20094227. https://doi.org/10.1101/2020.05.07.20094227

8. Almeida KMV. Assessment of functional health literacy in Brazilian careers of older people. Dement Neuropsychol. 2019;13(2):180-86. https://doi.org/10.1590/1980-57642018dn13-020006

9. Lima MFG, Vasconcelos EMR, Borba AKOT. Instrumentos utilizados para avaliar o letramento funcional em saúde de idosos com doença renal crônica: revisão integrativa. Rev Bras Geriatr Gerontol. 2019;22(3). https://doi. org/10.1590/1981-22562019022.180198

10. Mcculloch EC. Older Adults In: Parnell TA. Health Literacy in nursing: providing person-centered. Springer; 2015.320 p. 